\title{
Multiple Solutions for Nonlinear Coercive Problems with a Nonhomogeneous Differential Operator and a Nonsmooth Potential
}

\author{
Leszek Gasiński • Nikolaos S. Papageorgiou
}

Received: 30 August 2011 / Accepted: 30 September 2011/

Published online: 18 October 2011

(C) The Author(s) 2011. This article is published with open access at Springerlink.com

\begin{abstract}
We consider a nonlinear elliptic problem driven by a nonlinear nonhomogeneous differential operator and a nonsmooth potential. We prove two multiplicity theorems for problems with coercive energy functional. In both theorems we produce three nontrivial smooth solutions. In the second multiplicity theorem, we provide precise sign information for all three solutions (the first positive, the second negative and the third nodal). Out approach is variational, based on the nonsmooth critical point theory. We also prove an auxiliary result relating smooth and Sobolev local minimizer for a large class of locally Lipschitz functionals.
\end{abstract}

Keywords Locally Lipschitz function • Generalized subdifferential • Palais-Smale condition - Mountain pass theorem •

Second deformation theorem $\cdot$ Nodal solutions

Mathematics Subject Classifications (2010) $35 \mathrm{~J} 20 \cdot 35 \mathrm{~J} 70$

This research has been partially supported by the Ministry of Science and Higher Education of Poland under Grants no. N201 542438 and N201 604640.

\section{Gasiński $(\varangle)$}

Faculty of Mathematics and Computer Science,

Institute of Computer Science, Jagiellonian University,

ul. Łojasiewicza 6, 30-348 Kraków, Poland

e-mail: Leszek.Gasinski@ii.uj.edu.pl

\section{N. S. Papageorgiou}

Department of Mathematics, National Technical University,

Zografou Campus, Athens 15780, Greece

e-mail:npapg@math.ntua.gr 


\section{Introduction}

Let $\Omega \subseteq \mathbb{R}^{N}$ be a bounded domain with a $C^{2}$-boundary $\partial \Omega$. In this paper we study the following nonlinear elliptic problem with a nonsmooth potential (hemivariational inequality):

$$
\left\{\begin{array}{l}
-\operatorname{div} a(\nabla u(z)) \in \partial F(z, u(z)) \quad \text { in } \Omega \\
\left.u\right|_{\partial \Omega}=0
\end{array}\right.
$$

Here $a: \mathbb{R}^{N} \longrightarrow \mathbb{R}^{N}$ is a $C^{1}$-map, which is strictly monotone and satisfies certain other regularity conditions (see hypotheses $H_{0}^{\prime}$ ). Two important special cases of the map $a$ are the following:

$$
a(y)=\|y\|^{p-2} y \quad \forall y \in \mathbb{R}^{N}
$$

which corresponds to the $p$-Laplace differential operator

$$
\Delta_{p} u=\operatorname{div}\left(\|\nabla u\|^{p-2} \nabla u\right) \quad \forall u \in W_{0}^{1, p}(\Omega)
$$

and

$$
a(y)=\|y\|^{p-2} y+\mu\|y\|^{q-2} y \quad \forall y \in \mathbb{R}^{N},
$$

with $\mu \geqslant 0,2 \leqslant q \leqslant p<+\infty$, which corresponds to the $(p, q)$-differential operator

$$
\Delta_{p} u+\mu \Delta_{q} u, \quad \text { with } u \in W_{0}^{1, p}(\Omega) .
$$

Also $F: \Omega \times \mathbb{R} \longrightarrow \mathbb{R}$ is a measurable potential which is only locally Lipschitz and in general nonsmooth in the second variable. By $\partial F(z, \zeta)$ we denote the generalized (Clarke) subdifferential of $\zeta \longmapsto F(z, \zeta)$ (see Section 2).

We are interested in the existence of multiple nontrivial solutions for problem (1.1), when the energy functional of the problem is coercive. We prove two such multiplicity theorems ("three solutions theorems"). In the first, we produce three nontrivial smooth solutions, two of which have constant sign (one positive and the other negative). In the second multiplicity theorem, by strengthening the hypotheses on the potential $F(z, \cdot)$, we show that the third solution is nodal (sign changing). To the best of our knowledge this is the first result (even for smooth problems, i.e., when $F(z, \cdot) \in C^{1}(\mathbb{R})$ ), which produces a nodal solution for problems with a nonhomogeneous differential operator.

Our approach is variational based on the nonsmooth critical point theory (see Gasiński-Papageorgiou [18] and Motreanu-Rădulescu [31]). We mention that three solutions theorems for coercive equations were proved by Ambrosetti-Lupo [2], Ambrosetti-Mancini [3], Iannizzotto [24], Struwe [34] for certain parametric semilinear equations (Iannizzotto [24] deals with hemivariational inequalities, while the other consider "smooth" problems) and by Averna-Marano-Motreanu [4], Liu-Liu [27], Liu [28], Papageorgiou-Papageorgiou [33] for problems driven by $p$-Laplacian (Averna-Marano-Motreanu [4] deal with parametric hemivariational inequalities, while the others examine "smooth" potentials). Our work here is closer to those of Liu-Liu [27] and Liu [28], since no parameter appears in (1.1) and our theorems extend the results of [27] and [28] in many different ways. In the next three papers of Filippakis-Gasiński-Papageorgiou [14] and Gasiński-Papageorgiou $[16,17]$ we also find multiplicity results for hemivariational inequalities in the case 
of various boundary value conditions: Dirichlet, periodic and Neumann. Finally, we mention that hemivariational inequalities arise naturally in problems of nonsmooth mechanics. For several such applications, we refer to the book of NaniewiczPanagiotopoulos [32].

In the next section, for the convenience of the reader, we recall some basic facts from the nonsmooth critical point theory, which is based on the notion of subdifferential of a locally Lipschitz functional. We also prove an auxiliary result of independent interest relating smooth and Sobolev local minimizers for a large class of nonsmooth locally Lipschitz functions.

\section{Mathematical Background-Preliminary Results}

Let $X$ be a Banach space and $X^{*}$ its topological dual. By $\langle\cdot, \cdot\rangle$ we denote the duality brackets for the pair $\left(X^{*}, X\right)$. For a given locally Lipschitz functional $\varphi: X \longrightarrow \mathbb{R}$, the generalized directional derivative $\varphi^{0}(z ; h)$ of $\varphi$ at $x \in X$ in the direction $h \in X$, is defined by

$$
\varphi^{0}(x ; h) \stackrel{d f}{=} \limsup _{\substack{x^{\prime} \\ t \\ t \succ 0}} \frac{\varphi\left(x^{\prime}+t h\right)-\varphi\left(x^{\prime}\right)}{t} .
$$

It is easy to see that the map $x \longmapsto \varphi^{0}(x ; h)$ is sublinear continuous. Therefore, it is the support function of a nonempty, convex and $w^{*}$-compact set $\partial \varphi(x) \subseteq X^{*}$, defined by

$$
\partial \varphi(x)=\left\{x^{*} \in X^{*}:\left\langle x^{*}, h\right\rangle \leqslant \varphi^{0}(x ; h) \text { for all } h \in X\right\} .
$$

The multifunction $x \longmapsto \partial \varphi(x)$ is called the generalized (or Clarke) subdifferential of $\varphi$. If $\varphi: X \longrightarrow \mathbb{R}$ is continuous convex, then $\varphi$ is locally Lipschitz and the generalized subdifferential of $\varphi$ coincides with the subdifferential in the sense of convex analysis, given by

$$
\partial_{c} \varphi(x)=\left\{x^{*} \in X^{*}:\left\langle x^{*}, h\right\rangle \leqslant \varphi(x+h)-\varphi(h) \text { for all } h \in X\right\} .
$$

Moreover, if $\varphi \in C^{1}(X)$, then $\varphi$ is locally Lipschitz and $\partial \varphi(x)=\left\{\varphi^{\prime}(x)\right\}$.

If $\varphi, \psi: X \longrightarrow \mathbb{R}$ are locally Lipschitz functionals and $\lambda \in \mathbb{R}$, then

$$
\partial(\varphi+\psi)(x) \subseteq \partial \varphi(x)+\partial \psi(x) \quad \forall x \in X
$$

and

$$
\partial(\lambda \varphi)(x)=\lambda \partial \varphi(x) \quad \forall x \in X, \lambda \in \mathbb{R} .
$$

The generalized subdifferential has a very rich calculus, which extends that of smooth and of continuous convex functionals. For more details, we refer to the book of Clarke [9].

Let $\varphi: X \longrightarrow \mathbb{R}$ be a locally Lipschitz functional. We say that $x \in X$ is a critical point of $\varphi$, if $0 \in \partial \varphi(x)$. If $x \in X$ is a local extremum of $\varphi$ (i.e., $x$ is either a local minimizer or a local maximizer of $\varphi$ ), then $x \in X$ is a critical point of $\varphi$.

For a given locally Lipschitz functional $\varphi: X \longrightarrow \mathbb{R}$, we set

$$
m_{\varphi}(x)=\inf \left\{\left\|x^{*}\right\|_{*}: x^{*} \in \partial \varphi(x)\right\}
$$


(here $\|\cdot\|_{*}$ denotes the norm of the dual space $X^{*}$ ). We say that $\varphi$ satisfies the Palais-Smale condition, if the following holds:

Every sequence $\left\{x_{n}\right\}_{n \geqslant 1} \subseteq X$, such that $\left\{\varphi\left(x_{n}\right)\right\}_{n \geqslant 1} \subseteq \mathbb{R}$ is a bounded sequence and

$$
m_{\varphi}\left(x_{n}\right) \longrightarrow 0
$$

admits a strongly convergent subsequence.

Using this compactness-type condition, we can have the following nonsmooth extension of the well known mountain pass theorem.

Theorem 2.1 If $X$ is a Banach space, $\varphi: X \longrightarrow \mathbb{R}$ is a locally Lipschitz functional which satisfies the Palais-Smale condition, $x_{0}, x_{1} \in X$ are such that $\left\|x_{1}-x_{0}\right\|>r>0$,

$$
\max \left\{\varphi\left(x_{0}\right), \varphi\left(x_{1}\right)\right\}<\inf \left\{\varphi(x):\left\|x-x_{0}\right\|=r\right\}=\eta_{0}
$$

and

$$
c=\inf _{\gamma \in \Gamma} \max _{0 \leqslant t \leqslant 1} \varphi(\gamma(t))
$$

where

$$
\Gamma=\left\{\gamma \in C([0,1] ; X): \gamma(0)=x_{0}, \gamma(1)=x_{1}\right\},
$$

then $c \geqslant \eta_{0}$ and $c$ is a critical value of the functional $\varphi$.

The nonsmooth critical point theory was initiated with the work of Chang [6]. Detailed presentations of the theory with extensions and generalizations can be found in the books of Gasiński-Papageorgiou [18] and Motreanu-Rădulescu [31].

Let $\varphi: X \longrightarrow \mathbb{R}$ be a locally Lipschitz functional and $c \in \mathbb{R}$. We define

$$
\begin{aligned}
\dot{\varphi}^{c} & =\{x \in X: \varphi(x)<c\}, \\
K_{\varphi} & =\{x \in X: 0 \in \partial \varphi(x)\}, \\
K_{\varphi}^{c} & =\left\{x \in K_{\varphi}: \varphi(x)=c\right\} .
\end{aligned}
$$

The next result is due to Corvellec [10] and it is the nonsmooth counterpart of the so called second deformation theorem (see Gasiński-Papageorgiou [19, p. 628]).

Theorem 2.2 If $X$ is a Banach space, $\varphi: X \longrightarrow \mathbb{R}$ is a locally Lipschitz functional which satisfies the Palais-Smale condition, $a \in \mathbb{R}, b \in \mathbb{R} \cup\{+\infty\}, K_{\varphi} \cap \varphi^{-1}(a, b)=\emptyset$ and $K_{\varphi}^{c}$ is finite and contains only local minimizers of $\varphi$, then there exists a continuous deformation $h:[0,1] \times \dot{\varphi}^{b} \longrightarrow \dot{\varphi}^{b}$, such that:

(a) $\left.h(t, \cdot)\right|_{K_{\varphi}^{a}}=\left.i d\right|_{K_{\varphi}^{a}}$ for all $t \in[0,1]$;

(b) $h\left(1, \dot{\varphi}^{b}\right) \subseteq \dot{\varphi}^{a} \cup K_{\varphi}^{a}$

(c) $\varphi(h(t, x)) \leqslant \varphi(x)$ for all $(t, x) \in[0,1] \times \dot{\varphi}^{b}$.

In particular $\dot{\varphi}^{a} \cup K_{\varphi}^{a}$ is a weak deformation retract of $\dot{\varphi}^{b}$. 
In the analysis of problem (1.1), in addition to the Sobolev space $W_{0}^{1, p}(\Omega)$, we will also use the Banach space

$$
C_{0}^{1}(\bar{\Omega})=\left\{u \in C^{1}(\bar{\Omega}):\left.u\right|_{\partial \Omega}=0\right\} .
$$

This is an ordered Banach space with positive cone

$$
C_{+}=\left\{u \in C_{0}^{1}(\bar{\Omega}): u(z) \geqslant 0 \text { for all } z \in \bar{\Omega}\right\} .
$$

This cone has a nonempty interior, given by

$$
\text { int } C_{+}=\left\{u \in C_{+}: u(z)>0 \text { for all } z \in \Omega, \frac{\partial u}{\partial n}(z)<0 \text { for all } z \in \partial \Omega\right\},
$$

where $n(\cdot)$ denotes the outward unit normal on $\partial \Omega$.

For the next auxiliary result, we can be more general and allow the map $a(\cdot)$ to be $z$-dependent. More precisely, we introduce the following hypotheses:

$\underline{H_{0}}: G: \bar{\Omega} \times \mathbb{R}^{N} \longrightarrow \mathbb{R}$ is a $C^{1}$-function, such that $G(z, 0)=0, \nabla_{y} G(z, y)=a(z, y)$ and $\bar{a}(z, 0)=0$ for almost all $z \in \Omega$ and

(i) $\quad a \in C^{1}\left(\bar{\Omega} \times\left(\mathbb{R}^{N} \backslash\{0\}\right) ; \mathbb{R}^{N}\right)$;

(ii) there exist $c_{0}>0$ and $\eta \geqslant 0$, such that for every $z \in \bar{\Omega}$ and every $y \in \mathbb{R}^{N} \backslash\{0\}$, we have

$$
c_{0}(\eta+\|y\|)^{p-2}\|\xi\|^{2} \leqslant\left(\nabla_{y} a(z, y) \xi, \xi\right)_{\mathbb{R}^{N}} \quad \forall \xi \in \mathbb{R}^{N}
$$

(iii) there exists $c_{1}>0$, such that for every $z \in \bar{\Omega}$ and every $y \in \mathbb{R}^{N} \backslash\{0\}$, we have

$$
\left\|\nabla_{y} a(z, y)\right\| \leqslant c_{1}(\eta+\|y\|)^{p-2},
$$

with $\eta$ as in (ii);

(iv) for every $\varrho>0$, there exists $c_{2}=c_{2}(\varrho)>0$, such that

$$
\left|a(z, y)-a\left(z^{\prime}, y\right)\right| \leqslant c_{2}(1+\|y\|)^{p-1}\left\|z-z^{\prime}\right\| \quad \forall z \in \Omega, z^{\prime} \in \partial \Omega,\|y\| \leqslant \varrho .
$$

Example 2.3 The following maps satisfy hypotheses $H_{0}$ :

(a) Let

$$
G_{1}(z, y)=\frac{1}{p} \vartheta(z)\|y\|^{p},
$$

with $\vartheta \in C^{1}(\bar{\Omega}), \vartheta(z)>0$ for all $z \in \bar{\Omega}$ and $1<p<+\infty$. Then

$$
a_{1}(z, y)=\vartheta(z)\|y\|^{p-2} y .
$$

This potential function corresponds to a weighted $p$-Laplacian differential operator.

(b) Let

$$
G_{2}(z, y)=\frac{\vartheta_{1}(z)}{p}\|y\|^{p}+\frac{\vartheta_{2}(z)}{q}\|y\|^{q},
$$

with $\vartheta_{1}, \vartheta_{2} \in C^{1}(\bar{\Omega}), \vartheta_{1}(z)>0, \vartheta_{2}(z)>0$ for all $z \in \bar{\Omega}$ and $2 \leqslant q \leqslant p<+\infty$. Then

$$
a_{2}(z, y)=\vartheta_{1}(z)\|y\|^{p-2} y+\vartheta_{2}(z)\|y\|^{q-2} y .
$$


This potential function corresponds to a weighted $(p, q)$-differential operator.

Problems with such potentials were studied recently by Cingolani-Degiovanni (c) Let

[7], Figueiredo [13], Medeiros-Perera [29].

$$
G_{3}(z, y)=\frac{\vartheta(z)}{p}\left(\|y\|^{p}+\ln \left(1+\|y\|^{p}\right)\right),
$$

with $\vartheta \in C^{1}(\bar{\Omega}), \vartheta(z)>0$ for all $z \in \bar{\Omega}$ and $p \geqslant 2$. Then

$$
a_{3}(z, y)=\vartheta(z)\left(\|y\|^{p-2} y+\frac{\|y\|^{p-2} y}{1+\|y\|^{2}}\right) \text {. }
$$

From hypotheses $H_{0}$ and using the integral form of the mean value theorem, we obtain the following auxiliary result.

Lemma 2.4 If hypotheses $H_{0}$ hold, then for all $z \in \bar{\Omega}, a(z, \cdot)$ is strictly monotone and for all $(z, y) \in \bar{\Omega} \times \mathbb{R}^{N}$, we have

$$
(a(z, y), y)_{\mathbb{R}^{N}} \geqslant \frac{c_{0}}{p-1}\|y\|^{p} \quad \text { and } \quad\|a(z, y)\| \leqslant c_{1}(\eta+\|y\|)^{p-1} .
$$

An easy consequence of this lemma are the following growth estimates for the potential $G(z, \cdot)$.

Corollary 2.5 If hypotheses $H_{0}$ hold, then for all $z \in \bar{\Omega}, G(z, \cdot)$ is strictly convex and

$$
\frac{c_{0}}{p(p-1)}\|y\|^{p} \leqslant G(z, y) \leqslant \hat{c}_{1}(1+\|y\|)^{p} \quad \forall(z, y) \in \bar{\Omega} \times \mathbb{R}^{N} .
$$

The next result relates local $C_{0}^{1}(\bar{\Omega})$ and $W_{0}^{1, p}(\Omega)$-minimizers for a large class of locally Lipschitz functionals. Such a result was first proved for

$$
G(y)=\frac{1}{2}\|y\|^{2}
$$

and smooth (i.e., $C^{1}$ ) functionals by Brézis-Nirenberg [5]. It was extended to the case

$$
G(y)=\frac{1}{p}\|y\|^{p},
$$

with $1<p<+\infty$ and smooth functionals by García Azorero-Manfredi-Peral Alonso [15] (see also Guo-Zhang [21], where $p \geqslant 2$ ). For a nonsmooth version we refer to Gasiński-Papageorgiou [18, p. 655]. The next proposition extends all the aforementioned works. Moreover our proof is simpler.

So, let $F_{0}: \Omega \times \mathbb{R} \longrightarrow \mathbb{R}$ be a measurable function, such that for almost all $z \in \Omega$, the function $\zeta \longmapsto F_{0}(z, \zeta)$ is locally Lipschitz and

$$
|u| \leqslant a(z)+c|\zeta|^{r-1} \quad \text { for a.a. } z \in \Omega \text {, all } \zeta \in \mathbb{R} \text {, all } u \in \partial F_{0}(z, \zeta),
$$

with $a \in L^{\infty}(\Omega)_{+}, c>0$ and $1<r<p^{*}$, where

$$
p^{*}= \begin{cases}\frac{N p}{N-p} & \text { if } p<N, \\ +\infty & \text { if } p \geqslant N .\end{cases}
$$


Let $\psi_{0}: W_{0}^{1, p}(\Omega) \longrightarrow \mathbb{R}$ be the functional, defined by

$$
\psi_{0}(u)=\int_{\Omega} G(z, \nabla u(z)) d z-\int_{\Omega} F_{0}(z, u(z)) d z \quad \forall u \in W_{0}^{1, p}(\Omega) .
$$

Evidently $\psi_{0}$ is Lipschitz continuous on bounded sets, hence it is locally Lipschitz.

Proposition 2.6 If hypotheses $H_{0}$ hold and $u_{0} \in W_{0}^{1, p}(\Omega)$ is a local $C_{0}^{1}(\bar{\Omega})$-minimizer of $\psi_{0}$, i.e., there exists $\varrho_{0}>0$, such that

$$
\psi_{0}\left(u_{0}\right) \leqslant \psi_{0}\left(u_{0}+h\right) \quad \forall h \in C_{0}^{1}(\bar{\Omega}),\|h\|_{C_{0}^{1}(\bar{\Omega})} \leqslant \varrho_{0},
$$

then $u_{0} \in C_{0}^{1, \beta}(\bar{\Omega})$ for some $\beta \in(0,1)$ and it is also a local $W_{0}^{1, p}(\Omega)$-minimizer of $\psi_{0}$, i.e., there exists $\varrho_{1}>0$, such that

$$
\psi_{0}\left(u_{0}\right) \leqslant \psi_{0}\left(u_{0}+h\right) \quad \forall h \in W_{0}^{1, p}(\Omega),\|h\| \leqslant \varrho_{1} .
$$

Proof Let $h \in C_{0}^{1}(\bar{\Omega})$ and consider $t>0$ small. Then by hypothesis

$$
\psi_{0}\left(u_{0}\right) \leqslant \psi_{0}\left(u_{0}+t h\right)
$$

so

$$
0 \leqslant \psi_{0}^{0}\left(u_{0} ; h\right)
$$

Since $h \in C_{0}^{1}(\bar{\Omega})$ is arbitrary, $\psi_{0}^{0}\left(u_{0} ; \cdot\right)$ is continuous and $C_{0}^{1}(\bar{\Omega})$ is dense in $W_{0}^{1, p}(\Omega)$, from (2.1), we infer that

$$
0 \leqslant \psi_{0}^{0}\left(u_{0} ; h\right) \quad \forall h \in W_{0}^{1, p}(\Omega)
$$

So

$$
0 \in \partial \psi_{0}\left(u_{0}\right)
$$

and thus

$$
V\left(u_{0}\right)=u^{*}
$$

where $V: W_{0}^{1, p}(\Omega) \longrightarrow W^{-1, p^{\prime}}(\Omega)=W_{0}^{1, p}(\Omega)^{*}$ (with $\left.\frac{1}{p}+\frac{1}{p^{\prime}}=1\right)$ is the nonlinear map, defined by

$$
\langle V(u), y\rangle=\int_{\Omega}(a(z, \nabla u), \nabla y)_{\mathbb{R}^{N}} d z \quad \forall u, y \in W_{0}^{1, p}(\Omega)
$$

and $u_{0}^{*} \in L^{r^{\prime}}(\Omega)$ (with $\left.\frac{1}{r}+\frac{1}{r^{\prime}}=1\right), u_{0}^{*}(z) \in \partial F_{0}\left(z, u_{0}(z)\right)$ for almost all $z \in \Omega$ (see Clarke [9, p. 83]). From (2.2), it follows that

$$
\left\{\begin{array}{l}
-\operatorname{div} a\left(z, \nabla u_{0}(z)\right)=u_{0}^{*}(z) \in \partial F_{0}\left(z, u_{0}(z)\right) \quad \text { in } \Omega, \\
\left.u_{0}\right|_{\partial \Omega}=0 .
\end{array}\right.
$$

Invoking Theorem 7.1 of Ladyzhenskaya-Uraltseva [25, p. 286], we have that $u_{0} \in$ $L^{\infty}(\Omega)$. Then on (2.3) we can use Theorem 1 of Lieberman [26] and conclude that $u_{0} \in C_{0}^{1, \beta}(\bar{\Omega})$ for some $\beta \in(0,1)$. 
Next we show that $u_{0}$ is also a local $W_{0}^{1, p}(\Omega)$-minimizer of $\psi_{0}$. We argue by contradiction. So, suppose that $u_{0}$ is not a local $W_{0}^{1, p}(\Omega)$-minimizer of $\psi_{0}$. For $\varepsilon>0$, let

$$
\bar{B}_{\varepsilon}^{r}=\left\{u \in W_{0}^{1, p}(\Omega):\|u\|_{r} \leqslant \varepsilon\right\}
$$

and consider the following minimization problem:

$$
\inf _{h \in \bar{B}_{\varepsilon}^{r}} \psi_{0}\left(u_{0}+h\right)=m_{0}^{\varepsilon}>-\infty
$$

Since $u_{0}$ is not a local $W_{0}^{1, p}(\Omega)$-minimizer of $\psi_{0}$, we have

$$
m_{0}^{\varepsilon}<\psi_{0}\left(u_{0}\right)
$$

Let $\left\{h_{n}\right\}_{n \geqslant 1} \subseteq \bar{B}_{\varepsilon}^{r}$ be a minimizing sequence for problem (2.4). Using Corollary 2.5 and the growth hypothesis on $\partial F_{0}(z, \cdot)$, we see that the sequence $\left\{h_{n}\right\}_{n \geqslant 1} \subseteq W_{0}^{1, p}(\Omega)$ is bounded. So, passing to a subsequence if necessary, we may assume that

$$
\begin{array}{ll}
h_{n} \longrightarrow h_{\varepsilon} \quad \text { weakly in } W_{0}^{1, p}(\Omega), \\
h_{n} \longrightarrow h_{\varepsilon} \quad \text { in } L^{r}(\Omega) .
\end{array}
$$

Clearly $\psi_{0}$ is sequentially weakly lower semicontinuous. So, from (2.6), we have

$$
\psi_{0}\left(u_{0}+h_{\varepsilon}\right) \leqslant \liminf _{n \rightarrow+\infty} \psi_{0}\left(u_{0}+h_{n}\right)
$$

hence

$$
\psi_{0}\left(u_{0}+h_{\varepsilon}\right)=m_{0}^{\varepsilon}
$$

and thus $h_{\varepsilon} \neq 0$ (see (2.5)).

So, the infimum in problem (2.4) is realized at some $h_{\varepsilon} \in \bar{B}_{\varepsilon}^{r} \backslash\{0\}$ (see (2.6)). Invoking the nonsmooth Lagrange multiplier rule of Clarke [8], we can find $\lambda_{\varepsilon} \leqslant 0$, such that

$$
0 \in \partial \psi_{0}\left(u_{0}+h_{\varepsilon}\right)-\lambda_{\varepsilon}\left|h_{\varepsilon}\right|^{r-2} h_{\varepsilon}
$$

so

$$
V\left(u_{0}+h_{\varepsilon}\right)=u_{\varepsilon}^{*}+\lambda_{\varepsilon}\left|h_{\varepsilon}\right|^{r-2} h_{\varepsilon}
$$

where $u_{\varepsilon}^{*} \in L^{r^{\prime}}(\Omega), u_{\varepsilon}^{*}(z) \in \partial F_{0}\left(z,\left(u_{0}+h_{\varepsilon}\right)(z)\right)$ for almost all $z \in \Omega$. Then

$$
\left\{\begin{array}{l}
-\operatorname{div} a\left(z, \nabla\left(u_{0}+h_{\varepsilon}\right)(z)\right)=u_{\varepsilon}^{*}(z)+\lambda_{\varepsilon}\left|h_{\varepsilon}(z)\right|^{r-2} h_{\varepsilon}(z) \text { in } \Omega, \\
\left.h_{\varepsilon}\right|_{\partial \Omega}=0 .
\end{array}\right.
$$

From (2.3) and (2.8), for almost all $z \in \Omega$, we have

$$
\begin{gathered}
-\operatorname{div}\left(a\left(z, \nabla\left(u_{0}+h_{\varepsilon}\right)(z)\right)-a\left(z, \nabla u_{0}(z)\right)\right) \\
=u_{\varepsilon}^{*}(z)-u_{0}^{*}(z)+\lambda_{\varepsilon}\left|h_{\varepsilon}(z)\right|^{r-2} h_{\varepsilon}(z)
\end{gathered}
$$


Case 1 Suppose that $\lambda_{\varepsilon} \in[-1,0]$ for all $\varepsilon \in(0,1]$.

We set

$$
\begin{aligned}
w_{\varepsilon}(z) & =\left(u_{0}+h_{\varepsilon}\right)(z), \\
\sigma_{\varepsilon}(z, y) & =a(z, y)-a\left(z, \nabla u_{0}(z)\right) .
\end{aligned}
$$

Then from (2.9), for almost all $z \in \Omega$, we have

$$
-\operatorname{div} \sigma_{\varepsilon}\left(z, \nabla w_{\varepsilon}(z)\right)=u_{\varepsilon}^{*}(z)-u_{0}^{*}(z)+\lambda_{\varepsilon}\left|\left(w_{\varepsilon}-u_{0}\right)(z)\right|^{r-2}\left(w_{\varepsilon}-u_{0}\right)(z) .
$$

On (2.10) we apply Theorem 7.1 of Ladyzhenskaya-Uraltseva [25, p. 286] and produce $M_{1}>0$, such that

$$
\left\|w_{\varepsilon}\right\|_{\infty} \leqslant M_{1} \quad \forall \varepsilon \in(0,1] .
$$

Clearly $\sigma_{\varepsilon}(z, y)$ satisfies hypotheses $H_{0}$. This fact and (2.11), permit the use of Theorem 1 of Lieberman [26] and so we can find $\gamma \in(0,1)$ and $M_{2}>0$, such that

$$
w_{\varepsilon} \in C_{0}^{1, \gamma}(\bar{\Omega}) \quad \text { and } \quad\left\|w_{\varepsilon}\right\|_{C_{0}^{1, \gamma}(\bar{\Omega})} \leqslant M_{2} \quad \forall \varepsilon \in(0,1]
$$

Case 2 Suppose that $\lambda_{\varepsilon_{n}}<-1$ for all $n \geqslant 1$, with $\varepsilon_{n} \searrow 0, \varepsilon_{n} \in(0,1]$ for all $n \geqslant 1$.

In this case we set

$$
\begin{aligned}
w_{\varepsilon_{n}} & =u_{0}+h_{\varepsilon_{n}} \\
\widehat{\sigma}_{\varepsilon_{n}}(z, y) & =\frac{1}{\left|\lambda_{\varepsilon_{n}}\right|}\left(a\left(z, \nabla u_{0}(z)+y\right)-a\left(z, \nabla u_{0}(z)\right)\right) .
\end{aligned}
$$

Then for almost all $z \in \Omega$ and all $n \geqslant 1$, we have

$$
-\operatorname{div} \widehat{\sigma}_{\varepsilon_{n}}\left(z, \nabla h_{\varepsilon_{n}}(z)\right)=\frac{1}{\left|\lambda_{\varepsilon_{n}}\right|}\left(u_{\varepsilon_{n}}^{*}(z)-u_{0}^{*}(z)\right)-\left|h_{\varepsilon_{n}}(z)\right|^{r-2} h_{\varepsilon_{n}}(z) .
$$

For every $\xi \in W_{0}^{1, p}(\Omega)$, we have

$$
\left\langle V\left(u_{0}\right), \xi\right\rangle=\int_{\Omega} u_{0}^{*} \xi d z
$$

(see (2.3)) and

$$
\left\langle V\left(u_{\varepsilon_{n}}\right), \xi\right\rangle=\int_{\Omega} u_{\varepsilon_{n}}^{*} \xi d z+\lambda_{\varepsilon_{n}} \int_{\Omega}\left|w_{\varepsilon_{n}}-u_{0}\right|^{r-2}\left(w_{\varepsilon_{n}}-u_{0}\right) \xi d z
$$

(see $(2.9))$.

Let $\mu \geqslant 1$ and consider the function $\left|w_{\varepsilon_{n}}-u_{0}\right|^{\mu}\left(w_{\varepsilon_{n}}-u_{0}\right)$. Then

$$
\nabla\left(\left|w_{\varepsilon_{n}}-u_{0}\right|^{\mu}\left(w_{\varepsilon_{n}}-u_{0}\right)\right)=(\mu+1)\left|w_{\varepsilon_{n}}-u_{0}\right|^{\mu} \nabla\left(w_{\varepsilon_{n}}-u_{0}\right),
$$

so

$$
\left|w_{\varepsilon_{n}}-u_{0}\right|^{\mu}\left(w_{\varepsilon_{n}}-u_{0}\right) \in W_{0}^{1, p}(\Omega) \quad \forall n \geqslant 1
$$

(recall that $w_{\varepsilon_{n}}, u_{0} \in C_{0}^{1}(\bar{\Omega})$ ). 
So, we can use this function as the test function $\xi$ in (2.14) and (2.15). We do this and then subtract (2.14) from (2.15). Using Lemma 2.4, for all $n \geqslant 1$ we obtain

$$
\begin{aligned}
0 \leqslant & (\mu+1) \int_{\Omega}\left(a\left(z, \nabla w_{\varepsilon_{n}}\right)-a\left(z, \nabla u_{0}\right), \nabla w_{\varepsilon_{n}}-\nabla u_{0}\right)_{\mathbb{R}^{N}}\left|w_{\varepsilon_{n}}-u_{0}\right|^{\mu} d z \\
= & \int_{\Omega}\left(u_{\varepsilon_{n}}^{*}-u_{0}^{*}\right)\left(w_{\varepsilon_{n}}-u_{0}\right)\left|w_{\varepsilon_{n}}-u_{0}\right|^{\mu} d z \\
& +\lambda_{\varepsilon_{n}} \int_{\Omega}\left|w_{\varepsilon_{n}}-u_{0}\right|^{r+\mu} d z .
\end{aligned}
$$

Because of (2.11), recalling that $u_{0} \in C_{0}^{1}(\bar{\Omega})$ and using Hölder inequality with exponents $\frac{\mu+r}{\mu+1}, \frac{\mu+r}{r-1}$, we have

$$
\begin{aligned}
& \left|\int_{\Omega}\left(u_{\varepsilon_{n}}^{*}-u_{0}^{*}\right)\left(w_{\varepsilon_{n}}-u_{0}\right)\right| w_{\varepsilon_{n}}-\left.u_{0}\right|^{\mu} d z \mid \\
& \quad \leqslant M_{3} \int_{\Omega}\left|w_{\varepsilon_{n}}-u_{0}\right|^{\mu+1} d z \\
& \quad \leqslant M_{3}|\Omega|_{N}^{\frac{r-1}{r+p}}\left\|w_{\varepsilon_{n}}-u_{0}\right\|_{r+\mu}^{\mu-1} \quad \forall n \geqslant 1,
\end{aligned}
$$

for some $M_{3}>0$. Here $|\cdot|_{N}$ stands for the Lebesgue measure on $\mathbb{R}^{N}$. We return to (2.16) and use (2.17). Then

$$
-\lambda_{\varepsilon_{n}}\left\|w_{\varepsilon_{n}}-u_{0}\right\|_{r+\mu}^{r+\mu} \leqslant M_{3}|\Omega|_{N}^{\frac{r-1}{r+\mu}}\left\|w_{\varepsilon_{n}}-u_{0}\right\|_{r+\mu}^{\mu-1}
$$

so

$$
-\lambda_{\varepsilon_{n}}\left\|w_{\varepsilon_{n}}-u_{0}\right\|_{r+\mu}^{r-1} \leqslant M_{3}|\Omega|_{N}^{\frac{r-1}{r+\mu}} \quad \forall \mu \geqslant 1, n \geqslant 1
$$

We let $\mu \rightarrow+\infty$ and obtain

$$
-\lambda_{\varepsilon_{n}}\left\|w_{\varepsilon_{n}}-u_{0}\right\|_{\infty}^{r-1} \leqslant M_{3} \quad \forall n \geqslant 1,
$$

So

$$
\left\|w_{\varepsilon_{n}}-u_{0}\right\|_{\infty}^{r-1} \leqslant \frac{M_{3}}{\left|\lambda_{\varepsilon_{n}}\right|} \quad \forall n \geqslant 1
$$

We return to (2.13) and denote the right hand side by $\eta_{\varepsilon_{n}}(z, \zeta)$. If $M_{4}=\left\|u_{0}\right\|+$ $M_{1}>0$ (see (2.11)), then for almost all $z \in \Omega$ and all $\zeta \in\left[-M_{4}, M_{4}\right]$, we have

$$
\left|\eta_{\varepsilon_{n}}(z, \zeta)\right| \leqslant \frac{1}{\left|\lambda_{\varepsilon_{n}}\right|}\left[M_{5}+M_{3}\right] \quad \forall n \geqslant 1,
$$

for some $M_{5}>0$. This fact and since $\widehat{\sigma}_{\varepsilon_{n}}(z, y)$ satisfies hypotheses $H_{0}$, permit the use of Theorem 1 of Lieberman [26] and so we can find $\gamma_{0} \in(0,1)$ and $M_{6}>0$, such that

$$
h_{\varepsilon_{n}} \in C_{0}^{1, \gamma_{0}}(\bar{\Omega}) \text { and }\left\|h_{\varepsilon_{n}}\right\|_{C_{0}^{1, \gamma_{0}}(\bar{\Omega})} \leqslant M_{6} \quad \forall n \geqslant 1
$$


Recall that for every $\gamma^{\prime} \in(0,1)$ the space $C_{0}^{1, \gamma^{\prime}}(\bar{\Omega})$ is embedded compactly in $C_{0}^{1}(\bar{\Omega})$. So, from (2.12) and (2.19) and by passing to a suitable subsequence if necessary, we have

$$
u_{0}+h_{\varepsilon_{n}} \longrightarrow u_{0} \quad \text { in } C_{0}^{1}(\bar{\Omega})
$$

(recall that $\varepsilon_{n} \searrow 0$ ). Because $u_{0}$ is a local $C_{0}^{1}(\bar{\Omega})$-minimizer of $\psi_{0}$, we can find $n_{0} \geqslant 1$, such that

$$
\psi_{0}\left(u_{0}\right) \leqslant \psi_{0}\left(u_{0}+h_{\varepsilon_{n}}\right) \quad \forall n \geqslant n_{0} .
$$

On the other hand since $h_{\varepsilon_{n}}$ are solutions of (2.4) and because of (2.5), we have

$$
\psi_{0}\left(u_{0}+h_{\varepsilon_{n}}\right)<\psi_{0}\left(u_{0}\right) \quad \forall n \geqslant 1 .
$$

Comparing (2.20) and (2.21), we reach a contradiction. This proves that $u_{0}$ is a local $W_{0}^{1, p}(\Omega)$-minimizer of $\psi_{0}$.

Recall (see the above proof) that $V: W_{0}^{1, p}(\Omega) \longrightarrow W^{-1, p^{\prime}}(\Omega)$ is the nonlinear map, defined by

$$
\langle V(u), y\rangle=\int_{\Omega}(a(z, \nabla u), \nabla y) d z \quad \forall u, y \in W_{0}^{1, p}(\Omega) .
$$

From Lemma 3.2 of Gasiński-Papageorgiou [20, p. 562], we have the following result.

Proposition 2.7 If hypotheses $H_{0}$ hold and $V: W_{0}^{1, p}(\Omega) \longrightarrow W^{-1, p^{\prime}}(\Omega)$ is defined by (2.22), then $V$ is continuous, bounded (i.e., maps bounded sets to bounded sets), strictly monotone and of type $(S)_{+}$, i.e., if $u_{n} \longrightarrow u$ weakly in $W_{0}^{1, p}(\Omega)$ and

$$
\limsup _{n \rightarrow+\infty}\left\langle V\left(u_{n}\right), u_{n}-u\right\rangle \leqslant 0,
$$

then $u_{n} \longrightarrow$ in $W_{0}^{1, p}(\Omega)$.

Let $\widehat{\lambda}_{1}$ be the first eigenvalue of $\left(-\Delta_{p}, W_{0}^{1, p}(\Omega)\right)$. We know that $\widehat{\lambda}_{1}>0$ is isolated, simple and

$$
\|\nabla u\|_{p}^{p} \geqslant \widehat{\lambda}_{1}\|u\|_{p}^{p} \quad \forall u \in W_{0}^{1, p}(\Omega)
$$

(see Gasiński-Papageorgiou [19]).

From Aizicovici-Papageorgiou-Staicu [1, Lemma 12], we have

Proposition 2.8 If $\vartheta \in L^{p}(\Omega)_{+}, \vartheta(z) \leqslant \frac{c_{0} \widehat{\lambda}_{1}}{p-1}$ for almost all $z \in \Omega, \vartheta \neq \frac{c_{0} \widehat{\lambda}_{1}}{p-1}$, then there exists $\xi_{0}>0$, such that

$$
\frac{c_{0}}{p-1}\|\nabla u\|_{p}^{p}-\int_{\Omega} \vartheta|u|^{p} d z \geqslant \xi_{0}\|u\|^{p} \quad \forall u \in W_{0}^{1, p}(\Omega) .
$$


Finally we mention that throughout this work, for every $u \in W_{0}^{1, p}(\Omega)$, we set

$$
\|u\|=\|\nabla u\|_{p}
$$

(by virtue of Poincaré inequality) and

$$
u^{+}=\max \{0, u\}, \quad u^{-}=\max \{0,-u\} .
$$

We know that $u^{+}, u^{-} \in W_{0}^{1, p}(\Omega)$ and $u=u^{+}-u^{-},|u|=u^{+}+u^{-}$. We mention that the notation $\|\cdot\|$ will also be used to denote the $\mathbb{R}^{N}$-norm. No confusion is possible, since it will always be clear from the context which norm is used. Also, as indicated in the proof of Proposition 2.6, $|\cdot|_{N}$ denotes the Lebesgue measure on $\mathbb{R}^{N}$.

\section{First Multiplicity Theorem}

In this section we prove a multiplicity theorem, which produces three nontrivial smooth solutions, two of which have constant sign (one positive, the other negative). To do this we need to drop the $z$-dependence on the map $a$. For easy reference, we state in detail the hypotheses:

$H_{0}^{\prime}: G: \mathbb{R}^{N} \longrightarrow \mathbb{R}$ is a $C^{1}$-function, such that $G(0)=0, \nabla G(y)=a(y)=a_{0}(\|y\|) y$, $a_{0}(\bar{t})>0, a(0)=0$ and

(i) $\quad a \in C^{1}\left(\mathbb{R}^{N} \backslash\{0\} ; \mathbb{R}^{N}\right)$

(ii) there exist $c_{0}>0$ and $\eta \geqslant 0$, such that for every $y \in \mathbb{R}^{N} \backslash\{0\}$, we have

$$
c_{0}(\eta+\|y\|)^{p-2}\|\xi\|^{2} \leqslant(\nabla a(y) \xi, \xi)_{\mathbb{R}^{N}} \quad \forall \xi \in \mathbb{R}^{N} ;
$$

(iii) there exists $c_{1}>0$, such that for every $y \in \mathbb{R}^{N} \backslash\{0\}$, we have

$$
\|\nabla a(y)\| \leqslant c_{1}(\eta+\|y\|)^{p-2},
$$

with $\eta$ as in (ii);

(iv) there exists $\tau \in(1, p)$, such that

$$
\lim _{y \rightarrow 0} \frac{G(y)}{\|y\|^{\tau}}=0 .
$$

Remark 3.1 Clearly, hypotheses $H_{0}^{\prime}$ are a particular case of hypotheses $H_{0}$. The reason we have dropped the $z$-dependence is that we need an extension of the nonlinear strong maximal principle of Vázquez [35], valid for the $p$-Laplacian, to more general nonhomogeneous differential operators, like the one in this paper. The only such result for $z$-dependent operators is that of Zhang [36], who though requires that $\eta=0$ in hypotheses $H_{0}$ (ii) and (iii). Such a condition excludes from consideration $(p, q)$-differential operators. Note that the examples presented after hypotheses $H_{0}$, satisfy hypotheses $H_{0}^{\prime}$ (of course with $\vartheta=\vartheta_{1}=\vartheta_{2}=1$ ).

The hypotheses on the nonlinear potential $F(z, \zeta)$ are the following:

$H_{1}: F: \Omega \times \mathbb{R} \longrightarrow \mathbb{R}$ is a measurable function, such that for almost all $z \in \Omega$, we have $F(z, 0)=0,0 \in \partial F(z, 0), F(z, \cdot)$ is locally Lipschitz and 
(i) there exist $a \in L^{\infty}(\Omega)_{+}$and $c>0$, such that

$$
\left|u^{*}\right| \leqslant a(z)+c|\zeta|^{p-1} \text { for almost all } z \in \Omega \text {, all } \zeta \in \mathbb{R} \text {, all } u^{*} \in \partial F(z, \zeta)
$$

(ii) there exists $\vartheta \in L^{\infty}(\Omega)_{+}, \vartheta(z) \leqslant \frac{c_{0}}{p-1}$ for almost all $z \in \Omega, \vartheta \neq \frac{c_{0}}{p-1}$, such that

$$
\limsup _{\zeta \rightarrow \pm \infty} \frac{p F(z, \zeta)}{|\zeta|^{p}} \leqslant \vartheta(z) \quad \text { uniformly for almost all } z \in \Omega
$$

(iii) if $\tau \in(1, p)$ is as in hypothesis $H_{0}^{\prime}(i v)$, then there exists $\beta_{0}>0$, such that

$$
\liminf _{\zeta \rightarrow 0} \frac{\tau F(z, \zeta)}{|\zeta|^{\tau}} \geqslant \beta_{0} \quad \text { uniformly for almost all } z \in \Omega
$$

(iv) for every $\varrho>0$, there exists $\gamma_{\varrho}>0$, such that, if

$$
\sigma(z, \zeta)=\min \left\{u^{*}: u^{*} \in \partial F(z, \zeta)\right\}
$$

then

$$
\sigma(z, \zeta)+\gamma_{\varrho}|\zeta|^{p-2} \zeta \geqslant 0 \text { for almost all } z \in \Omega \text {, all } \zeta \in[-\varrho, \varrho]
$$

Remark 3.2 Hypothesis $H_{1}(i i)$ implies that for almost all $z \in \Omega$, the function $F(z, \cdot)$ is $p$-(sub)linear near $\pm \infty$. Hypothesis $H_{1}$ (iii) implies the presence of a "concave" nonlinearity near the origin. We stress that no sign condition is imposed on the elements of $\partial F(z, \zeta)$. Instead, we impose the weaker condition $H_{1}(i v)$.

Example 3.3 The following potential function $F(\zeta)$ satisfies hypotheses $H_{1}$ (for the sake of simplicity, we drop the $z$-dependence):

$$
F(\zeta)=\left\{\begin{array}{l}
\frac{c}{\tau}|\zeta|^{\tau} \text { if }|\zeta| \leqslant 1, \\
\frac{\vartheta}{p}|\zeta|^{p} \text { if }|\zeta|>1,
\end{array}\right.
$$

with $1<\tau<p, c=\frac{\vartheta \tau}{p}$. Note that $F$ is not a $C^{1}$-function.

Let $\varphi: W_{0}^{1, p}(\Omega) \longrightarrow \mathbb{R}$ be the energy functional for problem (1.1), defined by

$$
\varphi(u)=\int_{\Omega} G(\nabla u(z)) d z-\int_{\Omega} F(z, u(z)) d z \quad \forall u \in W_{0}^{1, p}(\Omega) .
$$

Evidently $\varphi$ is locally Lipschitz.

Theorem 3.4 If hypotheses $H_{0}^{\prime}$ and $H_{1}$ hold, then problem (1.1) has at least three nontrivial smooth solutions:

$$
u_{0} \in \operatorname{int} C_{+}, \quad v_{0} \in-\operatorname{int} C_{+}, \quad \text { and } \quad y_{0} \in C_{0}^{1}(\bar{\Omega}) \backslash\{0\}
$$

and $u_{0}, v_{0}$ are local minimizers of $\varphi$. 
Proof Let

$$
F_{ \pm}(z, \zeta)=F\left(z, \pm \zeta^{ \pm}\right) \quad \forall(z, \zeta) \in \Omega \times \mathbb{R}
$$

and let $\varphi_{ \pm}: W_{0}^{1, p}(\Omega) \longrightarrow \mathbb{R}$ be the locally Lipschitz functionals, defined by

$$
\varphi_{ \pm}(u)=\int_{\Omega} G(\nabla u(z)) d z-\int_{\Omega} F_{ \pm}(z, u(z)) d z \quad \forall u \in W_{0}^{1, p}(\Omega) .
$$

By virtue of hypotheses $H_{1}(i)$ and (ii) and Lebourg mean value theorem for locally Lipschitz functionals (see e.g., Clarke [9, p. 41]), for a given $\varepsilon>0$, we can find $c_{3}=$ $c_{3}(\varepsilon)>0$, such that

$$
F(z, \zeta) \leqslant \frac{1}{p}(\vartheta(z)+\varepsilon)|\zeta|^{p}+c_{3} \text { for almost all } z \in \Omega \text {, all } \zeta \in \mathbb{R} .
$$

Then using Corollary 2.5, estimate (3.1) and Proposition 2.8, we have

$$
\begin{aligned}
\varphi_{+}(u) & =\int_{\Omega} G(\nabla u) d z-\int_{\Omega} F(z, u) d z \\
& \geqslant \frac{c_{0}}{p(p-1)}\|\nabla u\|_{p}^{p}-\frac{1}{p} \int_{\Omega} \vartheta|u|^{p} d z-\frac{\varepsilon}{p}\|u\|^{p}-c_{4} \\
& \geqslant \frac{1}{p}\left(\xi_{0}-\frac{\varepsilon}{\widehat{\lambda}_{1}}\right)\|u\|^{p}-c_{4} \quad \forall u \in W_{0}^{1, p}(\Omega) .
\end{aligned}
$$

Choosing $\varepsilon \in\left(0, \xi_{0} \widehat{\lambda}_{1}\right)$, we infer that $\varphi_{+}$is coercive. Also, exploiting the compactness of the embedding of $W_{0}^{1, p}(\Omega)$ into $L^{p}(\Omega)$, we can easily check that $\varphi_{+}$is sequentially weakly lower semicontinuous. So, by the Weierstrass theorem, we can find $u_{0} \in$ $W_{0}^{1, p}(\Omega)$, such that

$$
\varphi_{+}\left(u_{0}\right)=\inf _{u \in W_{0}^{1, p}(\Omega)} \varphi_{+}(u)=m_{+} .
$$

By virtue of hypotheses $H_{0}^{\prime}(\mathrm{iv})$ and $H_{1}(\mathrm{iii})$, for a given $\varepsilon>0$, we can find $\delta=\delta(\varepsilon)>0$ and $\beta_{1}=\beta_{1}(\varepsilon)>0$, such that for almost all $z \in \Omega$ and all $y \in \mathbb{R}^{N}, \zeta \in \mathbb{R}$ with $\|y\|<\delta$ and $|\zeta|<\delta$, we have

$$
G(y) \leqslant \frac{\varepsilon}{\tau}\|y\|^{\tau} \quad \text { and } \quad F(z, \zeta) \geqslant \frac{\beta_{1}}{\tau}|\zeta|^{\tau} .
$$

Let $u \in \operatorname{int} C_{+}$and let $t \in(0,1)$ be small, such that

$$
t u(z) \in[0, \delta] \text { and }\|\nabla(t u)(z)\| \in[0, \delta] \quad \forall z \in \bar{\Omega} .
$$

Then, using (3.3), we have

$$
\begin{aligned}
\varphi_{+}(t u) & =\int_{\Omega} G(\nabla(t u)) d z-\int_{\Omega} F(z, t u) d z \\
& \leqslant \frac{\varepsilon t^{\tau}}{\tau}\|\nabla u\|_{\tau}^{\tau}-\frac{\beta_{1} t^{\tau}}{\tau}\|u\|_{\tau}^{\tau} \\
& =\frac{t^{\tau}}{\tau}\left(\varepsilon\|u\|^{\tau}-\beta_{1}\|u\|_{\tau}^{\tau}\right) .
\end{aligned}
$$

Choosing $\varepsilon \in\left(0, \frac{\beta_{1}\|u\|_{\tau}^{\tau}}{\|u\|^{\tau}}\right)$, we see that

$$
\varphi_{+}(t u)<0,
$$


so

$$
\varphi_{+}\left(u_{0}\right)=m_{+}<0=\varphi_{+}(0)
$$

(see (3.2)), i.e., $u_{0} \neq 0$.

From (3.2), we have

$$
0 \in \partial \varphi_{+}\left(u_{0}\right)
$$

so

$$
V\left(u_{0}\right)=u_{0}^{*},
$$

where $u_{0}^{*} \in L^{p^{\prime}}(\Omega), u_{0}^{*} \in \partial F_{+}\left(z, u_{0}(z)\right)$ for almost all $z \in \Omega$.

From the nonsmooth chain rule (see Clarke [9, p. 42]), we have

$$
\partial F_{+}(z, \zeta) \subseteq \begin{cases}0 & \text { if } \zeta<0 \\ \{\xi \partial F(z, 0): \xi \in[0,1]\} & \text { if } 0 \leqslant \zeta \leqslant 1 \\ \partial F(z, \zeta) & \text { if } 1<\zeta\end{cases}
$$

On (3.3) we act with $-u_{0}^{-} \in W_{0}^{1, p}(\Omega)$ and using (3.5) and Lemma 2.4, we obtain

$$
\frac{c_{0}}{p-1}\left\|\nabla u_{0}^{-}\right\|_{p}^{p} \leqslant 0
$$

i.e., $u_{0} \geqslant 0, u_{0} \neq 0$.

From (3.4), we have

$$
\left\{\begin{array}{l}
-\operatorname{div} a\left(\nabla u_{0}(z)\right)=u_{0}^{*}(z) \quad \text { in } \Omega \\
\left.u_{0}\right|_{\partial \Omega}=0
\end{array}\right.
$$

Since

$$
\nabla u_{0}(z)=0 \quad \text { on }\left\{u_{0}=0\right\}
$$

(Stampacchia theorem; see e.g., Gasiński-Papageorgiou [19, p. 195]), we infer that

$$
u_{0}^{*}(z) \in \partial F\left(z, u_{0}(z)\right) \quad \text { for almost all } z \in \Omega
$$

(see (3.5)). So, $u_{0}$ is a nontrivial positive solution of problem (1.1). Moreover, as before (see the proof of Proposition 2.6), from the nonlinear regularity theory (see Ladyzhenskaya-Uraltseva [25] and Lieberman [26]), we have that $u_{0} \in C_{+} \backslash\{0\}$. Let $\varrho=\left\|u_{0}\right\|_{\infty}$ and let $\gamma_{\varrho}>0$ be as postulated by hypothesis $H_{1}(i v)$. Then

$$
-\operatorname{div} a\left(\nabla u_{0}(z)\right)+\gamma_{\varrho} u_{0}(z)^{p-1}=u_{0}^{*}(z)+\gamma_{\varrho} u_{0}(z)^{p-1} \geqslant 0 \quad \text { for almost all } z \in \Omega,
$$

so

$$
\operatorname{div} a\left(\nabla u_{0}(z)\right) \leqslant \gamma_{\varrho} u_{0}(z)^{p-1} \quad \text { for almost all } z \in \Omega
$$

and thus $u_{0} \in \operatorname{int} C_{+}$(see Montenegro [30, Theorem 6]).

If

$$
W_{+}=\left\{u \in W_{0}^{1, p}(\Omega): u(z) \geqslant 0 \text { for almost all } z \in \Omega\right\}
$$

then clearly

$$
\left.\varphi\right|_{W_{+}}=\left.\varphi_{+}\right|_{W_{+}} .
$$


So, $u_{0}$ is a local $C_{0}^{1}(\bar{\Omega})$-minimizer of $\varphi$. Invoking Proposition 2.6, we infer that $u_{0}$ is a local $W_{0}^{1, p}(\Omega)$-minimizer of $\varphi$.

Similarly, working with the functional $\varphi_{-}$, we produce one more constant sign smooth solution $v_{0} \in-\operatorname{int} C_{+}$of problem (1.1), which is a local minimizer of the functional $\varphi$.

Without any loss of generality, we may assume that

$$
\varphi\left(v_{0}\right) \leqslant \varphi\left(u_{0}\right)
$$

(the analysis is similar, if the opposite inequality is true) and that the set $K_{\varphi}$ is finite (otherwise, we already have infinity solutions for problem (1.1)). Reasoning as in Aizicovici-Papageorgiou-Staicu [1, proof of Proposition 29] (see also GasińskiPapageorgiou [20, Theorem 3.4]), we can find $\varrho \in(0,1)$ small, such that

$$
\varphi\left(v_{0}\right) \leqslant \varphi\left(u_{0}\right)<\inf \left\{\varphi(u):\left\|u-u_{0}\right\|=\varrho\right\}=\eta_{\varrho}, \quad\left\|v_{0}-u_{0}\right\|>\varrho .
$$

As we did for $\varphi_{+}$, in a similar way, using hypothesis $H_{1}(i i)$, we can check that $\varphi$ is coercive and so it satisfies the Palais-Smale condition. This fact, together with (3.6) permit the use of the nonsmooth mountain pass theorem (see Theorem 2.1). So, we can find $y_{0} \in W_{0}^{1, p}(\Omega)$, such that

$$
\varphi\left(v_{0}\right) \leqslant \varphi\left(u_{0}\right)<\eta_{\varrho} \leqslant \varphi\left(y_{0}\right)
$$

and

$$
0 \in \partial \varphi\left(y_{0}\right) .
$$

From (3.7) it is clear that $y_{0} \notin\left\{v_{0}, u_{0}\right\}$, while from (3.8), we have

$$
V\left(y_{0}\right)=\widehat{u}_{0}^{*},
$$

where $\widehat{u}_{0}^{*} \in L^{p^{\prime}}(\Omega), \widehat{u}_{0}^{*}(z) \in \partial F\left(z, y_{0}(z)\right)$ for almost all $z \in \Omega$. Hence $y_{0}$ is a solution of (1.1) and the nonlinear regularity theory implies that $y_{0} \in C_{0}^{1}(\bar{\Omega})$. It remains to show that $y_{0} \neq 0$. From Theorem 2.1, we have

$$
c=\varphi\left(y_{0}\right)=\inf _{\gamma \in \Gamma} \max _{0 \leqslant t \leqslant 1} \varphi(\gamma(t)),
$$

where

$$
\Gamma=\left\{\gamma \in C\left([0,1] ; W_{0}^{1, p}(\Omega)\right): \gamma(0)=v_{0}, \gamma(1)=u_{0}\right\} .
$$

From (3.9), we see that, if we can find $\gamma_{*} \in \Gamma$, such that

$$
\varphi\left(\gamma_{*}(t)\right)<0 \quad \forall t \in T
$$

then

$$
c=\varphi\left(y_{0}\right)<0=\varphi(0)
$$

and so $y_{0} \neq 0$. Hence our effort is on producing such a path $\gamma_{*} \in \Gamma$.

To this end, let

$$
\Gamma_{c}=\left\{\gamma \in C\left([0,1] ; C_{0}^{1}(\bar{\Omega})\right): \gamma(0)=v_{0}, \gamma(1)=u_{0}\right\} .
$$


By virtue of the density of the embedding of $C_{0}^{1}(\bar{\Omega})$ into $W_{0}^{1, p}(\Omega)$, we see that $\Gamma_{c}$ is dense in $\Gamma$. We can find $\widehat{\gamma} \in \Gamma_{c}$, such that $0 \notin \widehat{\gamma}([0,1])$. Since

$$
\widehat{\gamma}([0,1]) \subseteq C_{0}^{1}(\bar{\Omega}) \text { and } 0 \notin \widehat{\gamma}([0,1])
$$

we can find $\lambda \in(0,1)$ small, such that

$$
\lambda\|\nabla u(z)\| \leqslant \delta, \quad \text { and } \quad \lambda|u(z)| \leqslant \delta \quad \forall z \in \bar{\Omega}, u \in \widehat{\gamma}([0,1])
$$

(where $\delta$ is as in (3.3)) and

$$
\inf _{u \in \hat{\gamma}([0,1])}\|u\|_{\tau}^{\tau}=\widehat{m}>0
$$

For all $u \in \widehat{\gamma}([0,1])$, we have

$$
\begin{aligned}
\varphi(\lambda u) & =\int_{\Omega} G(\lambda \nabla u) d z-\int_{\Omega} F(z, \lambda u) d z \\
& \leqslant \lambda^{\tau} \varepsilon\|\nabla u\|_{\tau}^{\tau}-\beta_{1} \lambda^{\tau}\|u\|_{\tau}^{\tau} \\
& \leqslant \lambda^{\tau}\left(\varepsilon c_{5}-\beta_{1} \widehat{m}\right)
\end{aligned}
$$

for some $c_{5}>0$ (see (3.3), (3.10), (3.11) and note that $\widehat{\gamma}([0,1])$ is compact in $\left.W_{0}^{1, p}(\Omega)\right)$. Choosing $\varepsilon \in\left(0, \frac{\beta_{1} \widehat{m}}{c_{5}}\right)$ and setting $\tilde{\gamma}=\lambda \widehat{\gamma}$, from (3.12), we see that

$$
\left.\varphi\right|_{\tilde{\gamma}}<0
$$

and $\tilde{\gamma}$ is a continuous path in $W_{0}^{1, p}(\Omega)$ which connects $\lambda v_{0}$ and $\lambda u_{0}$.

Next, we will produce a continuous path in $W_{0}^{1, p}(\Omega)$ which connects $\lambda u_{0}$ and $u_{0}$ and along which $\varphi$ is strictly negative. To this end, recall that

$$
m_{+}=\inf _{u \in W_{0}^{1, p}(\Omega)} \varphi_{+}(u)<0=\varphi_{+}(0) .
$$

Also, we may assume that $K_{\varphi_{+}}^{m_{+}}=\left\{u_{0}\right\}$ or otherwise we already have a second positive solution (note that by virtue of (3.5) and the nonlinear regularity theory, $\left.K_{\varphi_{+}} \subseteq C_{+}\right)$. Invoking Theorem 2.2, we can find a continuous deformation $h:[0,1] \times$ $\dot{\varphi}_{+}^{0} \longrightarrow \dot{\varphi}_{+}^{0}$, such that

$$
h\left(1, \dot{\varphi}_{+}^{0}\right) \subseteq \dot{\varphi}_{+}^{m_{+}} \cup K_{\varphi_{+}}^{m_{+}}=\dot{\varphi}_{+}^{m_{+}} \cup\left\{u_{0}\right\}=\left\{u_{0}\right\}
$$

(since $\dot{\varphi}_{+}^{m_{+}}=\emptyset$ ) and

$$
\varphi_{+}(h(t, u)) \leqslant \varphi_{+}(u) \quad \forall t \in[0,1], u \in \dot{\varphi}_{+}^{0} .
$$

Consider the continuous path $\gamma_{+}:[0,1] \longrightarrow W_{0}^{1, p}(\Omega)$, defined by

$$
\gamma_{+}(t)=h\left(t, \lambda u_{0}\right)^{+} \quad \forall t \in[0,1]
$$

Then

$$
\gamma_{+}(0)=h\left(0, \lambda u_{0}\right)^{+}=\left(\lambda u_{0}\right)^{+}=\lambda u_{0}
$$


Also

$$
\gamma_{+}(1)=h\left(1, \lambda u_{0}\right)^{+}=u_{0}
$$

(see (3.14)). Hence $\gamma_{+}$is a continuous path in $W_{0}^{1, p}(\Omega)$ which connects $\lambda u_{0}$ and $u_{0}$. Moreover, from (3.15) and since $\left.\varphi\right|_{W_{+}}=\left.\varphi_{+}\right|_{W_{+}}$, we have

$$
\begin{aligned}
\varphi\left(\gamma_{+}(t)\right) & =\varphi\left(h\left(t, \lambda u_{0}\right)^{+}\right)=\varphi_{+}\left(h\left(t, \lambda u_{0}\right)^{+}\right) \\
& \leqslant \varphi_{+}\left(\lambda u_{0}\right)=\varphi\left(\lambda u_{0}\right)<0 \quad \forall t \in[0,1]
\end{aligned}
$$

$(\operatorname{see}(3.13))$, so

$$
\left.\varphi\right|_{\gamma_{+}}<0 \text {. }
$$

In a similar fashion, we produce a continuous path $\gamma_{-}$in $W_{0}^{1, p}(\Omega)$ which connects $\lambda v_{0}$ and $v_{0}$ and

$$
\left.\varphi\right|_{\gamma_{-}}<0 .
$$

We concatenate $\gamma_{-}, \tilde{\gamma}$ and $\gamma_{+}$and produce $\gamma_{*} \in \Gamma$, such that

$$
\left.\varphi\right|_{\gamma_{*}}<0
$$

(see (3.13), (3.16) and (3.17)), so $y_{0} \neq 0$ (see (3.9)).

So $y_{0} \in C_{0}^{1}(\bar{\Omega}) \backslash\{0\}$ is the third nontrivial smooth solution of (1.1).

\section{Second Multiplicity Theorem. Nodal Solutions}

In this section, we look for nodal solutions. To the best of our knowledge, there has been no previous work producing nodal solutions for equations driven by a nonhomogeneous differential operator. To do this, we need to strengthen the hypotheses on the nonsmooth potential $F(z, \zeta)$. For this purpose, let us first introduce some notation. Consider a measurable function $f: \Omega \times \mathbb{R} \longrightarrow \mathbb{R}$, such that for every $r>0$, we can find $a_{r} \in L^{\infty}(\Omega)_{+}$for which we have

$$
|f(z, \zeta)| \leqslant a_{r}(z) \text { for almost all } z \in \Omega \text { and all }|\zeta| \leqslant r .
$$

We allow $f(z, \cdot)$ to have jump discontinuities and define

$$
f_{l}(z, \zeta)=\liminf _{\zeta^{\prime} \rightarrow \zeta} f\left(z, \zeta^{\prime}\right) \text { and } f_{u}(z, \zeta)=\limsup _{\zeta^{\prime} \rightarrow \zeta} f\left(z, \zeta^{\prime}\right)
$$

For almost all $z \in \Omega$, these limits are finite. We assume that both $f_{l}$ and $f_{u}$ are superpositionally measurable. This means that, if $u: \Omega \longrightarrow \mathbb{R}$ is a measurable function, then so are functions $z \longmapsto f_{l}(z, u(z))$ and $z \longmapsto f_{u}(z, u(z))$.

We set

$$
F(z, \zeta)=\int_{0}^{\zeta} f(z, s) d s .
$$

Then for almost all $z \in \Omega$, the function $F(z, \cdot)$ is locally Lipschitz and

$$
\partial F(z, \zeta)=\left[f_{l}(z, \zeta), f_{u}(z, \zeta)\right]
$$

(see e.g., Chang [6] or Clarke [9]). We have $F(z, 0)=0$ for almost all $z \in \Omega$. 
So, now we can state precisely the new stronger conditions on the nonsmooth potential.

$\mathrm{H}_{2}: F: \Omega \times \mathbb{R} \longrightarrow \mathbb{R}$ is a function, such that

$$
F(z, \zeta)=\int_{0}^{\zeta} f(z, s) d s
$$

where $f: \Omega \times \mathbb{R} \longrightarrow \mathbb{R}$ is a measurable function satisfying:

(i) there exist $a \in L^{\infty}(\Omega)_{+}$and $c>0$, such that

$$
|f(z, \zeta)| \leqslant a(z)+c|\zeta|^{p-1} \text { for almost all } z \in \Omega \text {, all } \zeta \in \mathbb{R}
$$

the functions $f_{l}(z, \zeta)$ and $f_{u}(z, \zeta)$ defined by (4.1) are superpositionally measurable and for almost all $z \in \Omega$, the function $\zeta \longmapsto f(z, \zeta)$ is continuous at $\zeta=0$

(ii) there exists a function $\vartheta \in L^{\infty}(\Omega)_{+}, \vartheta(z) \leqslant \frac{c_{0}}{p-1}$ for almost all $z \in \Omega, \vartheta \neq \frac{c_{0}}{p-1}$, such that

$$
\limsup _{\zeta \rightarrow \pm \infty} \frac{p F(z, \zeta)}{|\zeta|^{p}} \leqslant \vartheta(z) \quad \text { uniformly for almost all } z \in \Omega
$$

(iii) there exist $\tau \in(1, p)$ and $c_{6}>0$, such that

$$
f_{l}(z, \zeta) \zeta \geqslant c_{6}|\zeta|^{\tau} \quad \text { for almost all } z \in \Omega \text { and all } \zeta \in \mathbb{R} \text {. }
$$

Remark 4.1 Note that the above hypotheses imply that

$$
\partial F(z, 0)=\{0\} \quad \text { for almost all } z \in \Omega .
$$

Also note that

$$
u^{*} \zeta \geqslant 0 \text { for almost all } z \in \Omega \text {, all } \zeta \in \mathbb{R} \text { and all } u^{*} \in \partial F(z, \zeta)
$$

( sign condition).

Example 4.2 The following potential function $\mathrm{F}$ satisfies hypotheses $\mathrm{H}_{2}$ :

$$
F(\zeta)=\frac{\vartheta}{p}|\zeta|^{p}+\max \left\{\frac{1}{\tau}|\zeta|^{\tau}, \frac{1}{q}|\zeta|^{q}\right\}+c|\zeta|,
$$

where $\vartheta<\frac{c_{0}}{p-1}, \tau, q \in(0, p)$ and $c \geqslant 0$. If $\tau \neq q$ and $c>0$, then $F$ is not a $C^{1}$ function.

First we show that problem (1.1) has extremal constant sign smooth solutions, i.e., there exists a smallest nontrivial positive solution and a biggest nontrivial negative solution.

$H_{0}^{\prime \prime}$ : Hypotheses $H_{0}^{\prime}$ hold and there exists $q \in(\tau, p)$, such that the map $t \mapsto G_{0}\left(t^{\frac{1}{q}}\right)$ is convex, with $G_{0}(t)=\int_{0}^{t} a_{0}(s) s d s$.

Proposition 4.3 If hypotheses $H_{0}^{\prime}$ and $H_{2}$ hold, then problem (1.1) has a smallest nontrivial positive solution $u_{+} \in \operatorname{int} C_{+}$and a biggest nontrivial negative solution $v_{-} \in-\operatorname{int} C_{+}$. 
Proof We consider the following auxiliary Dirichlet problem:

$$
\left\{\begin{array}{l}
-\operatorname{div} a(\nabla u(z))=c_{6}|u(z)|^{\tau-2} u(z) \quad \text { in } \Omega, \\
\left.u\right|_{\partial \Omega}=0 .
\end{array}\right.
$$

Claim Problem (4.2) has a unique nontrivial positive solution $\underline{u} \in \operatorname{int} C_{+}$and a unique nontrivial negative solution $\bar{v} \in-$ int $C_{+}$.

Let $\psi_{+}: W_{0}^{1, p}(\Omega) \longrightarrow \mathbb{R}$ be the $C^{1}$-functional, defined by

$$
\psi_{+}(u)=\int_{\Omega} G(\nabla u(z)) d z-\frac{c_{6}}{\tau}\left\|u^{+}\right\|_{\tau}^{\tau} \quad \forall u \in W_{0}^{1, p}(\Omega) .
$$

Using Corollary 2.5, we have

$$
\psi_{+}(u) \geqslant \frac{c_{0}}{p(p-1)}\|u\|^{p}-c_{7}\|u\|^{\tau} \quad \forall u \in W_{0}^{1, p}(\Omega),
$$

for some $c_{7}>0$.

Since $\tau<p$, from (4.3) we infer that $\psi_{+}$is coercive. Also, it is sequentially weakly lower semicontinuous. So, we can find $\underline{u} \in W_{0}^{1, p}(\Omega)$, such that

$$
\psi_{+}(\underline{u})=\inf _{u \in W_{0}^{1, p}(\Omega)} \psi_{+}(u)=\bar{m}_{+} .
$$

As in the proof of Theorem 3.4, using hypotheses $H_{0}^{1}(i v)$ and $H_{2}(i i i)$ and since $\tau<p$, we obtain

$$
\psi_{+}(\underline{u})=\bar{m}_{+}<0=\psi_{+}(0)
$$

$(\operatorname{see}(4.4))$, so

$$
\underline{u} \neq 0 \text {. }
$$

From (4.4), we have

$$
\psi_{+}^{\prime}(\underline{u})=0
$$

thus

$$
V(\underline{u})=c_{6}\left(\underline{u}^{+}\right)^{\tau-1} .
$$

Acting on (4.5) with $-\underline{u}^{-} \in W_{0}^{1, p}(\Omega)$ and using Lemma 2.4 , we obtain $\underline{u} \geqslant 0, \underline{u} \neq 0$. So, from (4.5), we have that $\underline{u}$ solves (4.2) and in fact the nonlinear regularity theory (see Ladyzhenskaya-Uraltseva [25] and Lieberman [26]) and the nonlinear maximum principle of Montenegro [30, Theorem 6], imply that $\underline{u} \in \operatorname{int} C_{+}$.

To show the uniqueness of the solution $\underline{u} \in$ int $C_{+}$, inspired by Diaz-Saa [11], we introduce the integral functional $\xi: W_{0}^{1, p}(\Omega) \longrightarrow \overline{\mathbb{R}}=\mathbb{R} \cup\{+\infty\}$, defined by

$$
\xi(u)= \begin{cases}\int_{\Omega} G\left(\nabla u^{\frac{1}{q}}\right) d z & \text { if } u \geqslant 0, u^{\frac{1}{q}} \in W_{0}^{1, q}(\Omega), \\ +\infty & \text { otherwise. }\end{cases}
$$

Clearly $\xi$ is convex, lower semicontinuous and it is not identical $+\infty$.

Let $u \in W_{0}^{1, p}(\Omega)$ be a nontrivial positive solution of the auxiliary problem (4.2). The nonlinear regularity theory (see $[25,26])$ and the nonlinear maximum principle 
(see [30]) imply that $u \in \operatorname{int} C_{+}$. Note that $u^{q} \geqslant 0$ and $\left(u^{q}\right)^{\frac{1}{q}} \in W_{0}^{1, q}(\Omega)$. So, $u^{q}$ is in the effective domain of the $\overline{\mathbb{R}}$-valued functional $\xi$. For $h \in C_{0}^{1}(\bar{\Omega})$ and $t>0$ small, we have $u^{q}+t h \in C_{+}$and so the directional derivative of $\xi$ at $u^{q}$ in the direction $h$ exists. Moreover, using the chain rule, we have

$$
\xi^{\prime}\left(u^{q}\right)(h)=\int_{\Omega} \frac{(G \circ D)^{\prime}(u)}{u^{q-1}} h d z=\int_{\Omega} \frac{-\operatorname{div} a(\nabla u)}{u^{q-1}} h d z .
$$

Let $\underline{w}$ be any nontrivial positive solution of (4.2). As above, we have that $\underline{w} \in$ int $C_{+}$. By virtue of the convexity of $\xi$ and (4.6), we have

$$
0 \leqslant \int_{\Omega}\left(-\frac{\operatorname{div} a(\nabla \underline{u})}{\underline{u}^{q-1}}+\frac{\operatorname{div} a(\nabla \underline{w})}{\underline{w}^{q-1}}\right)\left(\underline{u}^{q}-\underline{w}^{q}\right) d z,
$$

so

$$
0 \leqslant c_{6} \int_{\Omega}\left(\frac{1}{\underline{u}^{q-\tau}}-\frac{1}{\underline{w}^{q-\tau}}\right)\left(\underline{u}^{q}-\underline{w}^{q}\right) d z, \leqslant 0
$$

(see (4.2) and recall that $\tau<q$ ) and thus

$$
\underline{u}=\underline{w} .
$$

Similarly, we establish the uniqueness of the nontrivial negative solution $\bar{v} \in$ - int $C_{+}$. This proves the Claim.

Now, let $u$ be a nontrivial positive solution for problem (1.1). Such a solution exists by virtue of Theorem 3.4 and

$$
-\operatorname{div} a(\nabla u(z))=u^{*}(z) \text { for almost all } z \in \Omega,
$$

where $u^{*} \in L^{p^{\prime}}(\Omega), u^{*}(z) \in \partial F(z, u(z))$ for almost all $z \in \Omega$. It follows that $u \in \operatorname{int} C_{+}$ (see $[25,26])$. Let

$$
h_{+}(z, \zeta)= \begin{cases}0 & \text { if } \zeta<0 \\ c_{6} \zeta^{\tau-1} & \text { if } 0 \leqslant \zeta \leqslant u(z) \\ c_{6} u(z)^{\tau-1} & \text { if } u(z)<\zeta\end{cases}
$$

This is a Carathéodory function (i.e., for all $\zeta \in \mathbb{R}$, the function $z \longmapsto h_{+}(z, \zeta)$ is measurable and for almost all $z \in \Omega$, the function $\zeta \longmapsto h_{+}(z, \zeta)$ is continuous). We set

$$
H_{+}(z, \zeta)=\int_{0}^{\zeta} h_{+}(z, s) d s
$$

and consider the $C^{1}$-functional $\widehat{\psi}_{+}: W_{0}^{1, p}(\Omega) \longrightarrow \mathbb{R}$, defined by

$$
\widehat{\psi}_{+}(u)=\int_{\Omega} G(\nabla u(z)) d z-\int_{\Omega} H_{+}(z, u(z)) d z \quad \forall u \in W_{0}^{1, p}(\Omega) .
$$

It is clear from (4.8) and Corollary 2.5 that $\widehat{\psi}_{+}$is coercive. Also, it is sequentially weakly lower semicontinuous. So, we can find $\underline{\underline{u}} \in W_{0}^{1, p}(\Omega)$, such that

$$
\widehat{\psi}_{+}(\underline{\underline{u}})=\inf _{u \in W_{0}^{1, p}(\Omega)} \widehat{\psi}_{+}(u)=\widehat{m}_{+} .
$$


As before, since $\tau<p$, we have that

$$
\widehat{\psi}_{+}(\widehat{\widehat{u}})=\widehat{m}_{+}<0=\widehat{\psi}_{+}(0)
$$

(see (4.9)), i.e., $\underline{\widehat{u}} \neq 0$.

From (4.9), we have

$$
\widehat{\psi}_{+}^{\prime}(\widehat{\underline{u}})=0
$$

so

$$
V(\underline{\widehat{u}})=N_{h_{+}}(\underline{\widehat{u}}),
$$

where

$$
N_{h_{+}}(u)(\cdot)=h_{+}(\cdot, u(\cdot)) \quad \forall u \in W_{0}^{1, p}(\Omega) .
$$

Acting on (4.10) with $-\underline{u}^{-} \in W_{0}^{1, p}(\Omega)$, we obtain $\underline{\hat{u}} \geqslant 0, \widehat{\underline{u}} \neq 0$. On (4.10) we act also with $(\widehat{\underline{u}}-u)^{+} \in W_{0}^{1, p}(\Omega)$. Then

$$
\begin{aligned}
\left\langle V(\underline{\widehat{u}}),(\underline{\widehat{u}}-u)^{+}\right\rangle & =\int_{\Omega} h_{+}(z, \underline{\widehat{u}})(\widehat{\widehat{u}}-u)^{+} d z \\
& =\int_{\Omega} c_{6} u(z)^{\tau-1}(\widehat{\widehat{u}}-u)^{+} d z \\
& \leqslant \int_{\Omega} u^{*}(\widehat{\underline{u}}-u)^{+} d z \\
& =\left\langle V(u),(\underline{\widehat{u}}-u)^{+}\right\rangle
\end{aligned}
$$

(see (4.7) and hypothesis $H_{2}(i i i)$ ), so

$$
\int_{\{\underline{u}>u\}}(a(\nabla \underline{\widehat{u}})-a(\nabla u), \nabla \underline{\underline{u}}-\nabla u)_{\mathbb{R}^{N}} d z \leqslant 0,
$$

thus

$$
|\{\underline{\widehat{u}}>u\}|_{N}=0
$$

(see Lemma 2.4) and we obtain

$$
\widehat{\underline{u}} \leqslant u \text {. }
$$

So

$$
\widehat{\underline{u}} \in[0, u], \quad \widehat{\underline{u}} \neq 0,
$$

where

$$
[0, u]=\left\{w \in W_{0}^{1, p}(\Omega): 0 \leqslant w(z) \leqslant u(z) \text { for almost all } z \in \Omega\right\} .
$$

Then (4.10) becomes

$$
V(\underline{\widehat{u}})=c_{6} \widehat{\widehat{u}}^{\tau-1}
$$

(see (4.8)) and so

$$
\underline{\widehat{u}}=\underline{u} \in \operatorname{int} C_{+}
$$

(see the Claim). 
The above argument shows that

every nontrivial positive solution $u \in W_{0}^{1, p}(\Omega)$ of (1.1) satisfies $\underline{u} \leqslant u$.

A similar argument, using this time $\bar{v} \in-$ int $C_{+}$(see the Claim), shows that every nontrivial negative solution $v \in W_{0}^{1, p}(\Omega)$ of (1.1) satisfies $v \leqslant \bar{v}$.

Now we are ready to establish the existence of extremal nontrivial constant sign solutions of (1.1). So, let $S_{+}$be the set of nontrivial positive solutions of (1.1). From Theorem 3.4 we know that $S_{+} \neq \emptyset$. Let $C \subseteq S_{+}$be a chain (i.e., a total ordered subset of $S_{+}$). From Dunford-Schwartz [12, p. 336], we know that there exists a sequence $\left\{u_{n}\right\}_{n \geqslant 1} \subseteq C$, such that

$$
\inf _{n \geqslant 1} u_{n}=\inf C \text {. }
$$

Moreover, we can have the sequence $\left\{u_{n}\right\}_{n \geqslant 1}$ decreasing (see HeikkiläLakshmikantham [22, Lemma 1.1.5; p. 15]). We have

$$
V\left(u_{n}\right)=u_{n}^{*} \quad \forall n \geqslant 1,
$$

where $u_{n}^{*} \in L^{p^{\prime}}(\Omega)$ and $u_{n}^{*}(z) \in \partial F\left(z, u_{n}(z)\right)$ for almost all $z \in \Omega$. Evidently the sequence $\left\{u_{n}\right\}_{n \geqslant 1} \subseteq W_{0}^{1, p}(\Omega)$ is bounded (see (4.13) and recall that $u_{n} \leqslant u_{1}$ for all $n \geqslant 1)$. So, we may assume that

$$
\begin{array}{ll}
u_{n} \longrightarrow \tilde{u} & \text { weakly in } W_{0}^{1, p}(\Omega), \\
u_{n} \longrightarrow \tilde{u} & \text { in } L^{p}(\Omega) .
\end{array}
$$

On (4.13) we act with $u_{n}-\tilde{u}$, pass to the limit as $n \rightarrow+\infty$ and use (4.14). Then

$$
\lim _{n \rightarrow+\infty}\left\langle V\left(u_{n}\right), u_{n}-\widetilde{u}\right\rangle=0
$$

and so

$$
u_{n} \longrightarrow \tilde{u} \text { in } W_{0}^{1, p}(\Omega)
$$

(see Proposition 2.7). By virtue of hypothesis $H_{2}(i)$, the sequence $\left\{u_{n}^{*}\right\}_{n} \geqslant 1 \subseteq L^{p^{\prime}}(\Omega)$ is bounded and so, passing to a subsequence if necessary, we may assume that

$$
u_{n}^{*} \longrightarrow \widetilde{u}^{*} \text { weakly in } L^{p^{\prime}}(\Omega) \text {. }
$$

Invoking Proposition 3.9 of Hu-Papageorgiou [23, p. 694], we have

$$
\tilde{u}^{*}(z) \subseteq \text { conv } \limsup _{n \rightarrow+\infty} \partial F\left(z, u_{n}(z)\right) \subseteq \partial F(z, \widetilde{u}(z)) \quad \text { for almost all } z \in \Omega .
$$

So, if in (4.13) we pass to the limit as $n \rightarrow+\infty$ and use (4.16), we have

$$
V(\widetilde{u})=\tilde{u}^{*},
$$

where $\widetilde{u}^{*} \in L^{p^{\prime}}(\Omega), \widetilde{u}^{*}(z) \in \partial F(z, \widetilde{u}(z))$ for almost all $z \in \Omega$ and

$$
\tilde{u} \geqslant \underline{u}
$$

(see (4.11)). 
Therefore, we infer that $\tilde{u} \in S_{+}$and $\tilde{u} \in \inf C$. Since $C$ is an arbitrary chain, from the Kuratowski-Zorn lemma, we know that $S_{+}$has a minimal element $u_{+} \geqslant \underline{u}$, $u_{+} \in \operatorname{int} C_{+}$(from the nonlinear regularity theory). Using Lemma 3.2 of GasińskiPapageorgiou [20] (the lemma remains valid if the $p$-Laplacian is replaced by the more general differential operator $V$, since all we need is the monotonicity of $V$ ), we have that $S_{+}$is downward directed (i.e., if $u, w \in S_{+}$, then there exists $y \in S_{+}$, such that $y \leqslant \min \{u, w\})$. Therefore $u_{+} \in \operatorname{int} C_{+}$is the smallest positive solution of (1.1).

Similarly, we produce the biggest nontrivial negative solution $v_{-} \in-\operatorname{int} C_{+}$of problem (1.1) with $v_{-} \leqslant \bar{v}$ (see (4.12)) using this time Lemma 3.3 of GasińskiPapageorgiou [20].

Having these extremal solutions we can produce a nodal solution and have the second multiplicity theorem, which provides precise sign information for all three solutions.

Theorem 4.4 If hypotheses $H_{0}^{\prime \prime}$ and $H_{2}$ hold, then problem (1.1) has at least three nontrivial smooth solutions

$$
u_{0} \in \operatorname{int} C_{+}, \quad v_{0} \in-\operatorname{int} C_{+}, \quad \text { and } \quad y_{0} \in C_{0}^{1}(\bar{\Omega}) \backslash\{0\} \text { nodal } .
$$

Proof From Theorem 3.4 we already have two constant sign solutions

$$
u_{0} \in \operatorname{int} C_{+} \quad \text { and } \quad v_{0} \in-\operatorname{int} C_{+} .
$$

Let $u_{+} \in \operatorname{int} C_{+}$and $v_{-} \in-$ int $C_{+}$be the two extremal constant sign solutions of problem (1.1) postulated in Proposition 4.3. We have

$$
-\operatorname{div}\left(\nabla u_{+}(z)\right)=u_{+}^{*}(z) \text { for almost all } z \in \Omega,
$$

where $u_{+}^{*} \in L^{p^{\prime}}(\Omega), u_{+}^{*}(z) \in \partial F\left(z, u_{+}(z)\right)$ for almost all $z \in \Omega$, and

$$
-\operatorname{div}\left(\nabla v_{-}(z)\right)=v_{-}^{*}(z) \quad \text { for almost all } z \in \Omega,
$$

where $v_{-}^{*} \in L^{p^{\prime}}(\Omega), v_{-}^{*}(z) \in \partial F\left(z, v_{-}(z)\right)$ for almost all $z \in \Omega$.

We consider the following truncation of $f(z, \cdot)$ :

$$
\widehat{f}(z, \zeta)= \begin{cases}v_{-}^{*}(z) & \text { if } \zeta<v_{-}(z), \\ f(z, \zeta) & \text { if } v_{-}(z) \leqslant \zeta \leqslant u_{+}(z), \\ u_{+}^{*}(z) \text { if } u_{+}(z)<\zeta .\end{cases}
$$

Also, let

$$
\widehat{f_{ \pm}}(z, \zeta)=\widehat{f}\left(z, \pm \zeta^{ \pm}\right)
$$

We set

$$
\widehat{F}(z, \zeta)=\int_{0}^{\zeta} \widehat{f}(z, s) d s, \quad \widehat{F}_{ \pm}(z, \zeta)=\int_{0}^{\zeta} \widehat{f}_{ \pm}(z, s) d s
$$


and consider the locally Lipschitz functionals $\widehat{\varphi}, \widehat{\varphi}_{ \pm}: W_{0}^{1, p}(\Omega) \longrightarrow \mathbb{R}$, defined by

$$
\begin{aligned}
& \widehat{\varphi}(u)=\int_{\Omega} G(\nabla u(z)) d z-\int_{\Omega} \widehat{F}(z, u(z)) d z \quad \forall u \in W_{0}^{1, p}(\Omega), \\
& \widehat{\varphi}_{ \pm}(u)=\int_{\Omega} G(\nabla u(z)) d z-\int_{\Omega} \widehat{F}_{ \pm}(z, u(z)) d z \quad \forall u \in W_{0}^{1, p}(\Omega) .
\end{aligned}
$$

As in the proof of Proposition 4.3, we show that

$$
K_{\widehat{\varphi}} \subseteq\left[v_{-}, u_{+}\right], \quad K_{\widehat{\varphi}_{+}}=\left\{0, u_{+}\right\}, \quad K_{\widehat{\varphi}_{-}}=\left\{v_{-}, 0\right\} .
$$

Claim $u_{+}$and $v_{-}$are local minimizers of the functional $\widehat{\varphi}$.

Evidently $\widehat{\varphi}_{+}$is coercive (see (4.17)) and sequentially weakly lower semicontinuous. So, we can find $\widehat{u}_{+} \in W_{0}^{1, p}(\Omega)$, such that

$$
\widehat{\varphi}_{+}\left(\widehat{u}_{+}\right)=\inf _{u \in W_{0}^{1, p}(\Omega)} \widehat{\varphi}_{+}(u)=\widehat{m}_{+} .
$$

From hypothesis $H_{2}(i i i)$ and since $\tau<p$, we have

$$
\widehat{\varphi}_{+}\left(\widehat{u}_{+}\right)=\widehat{m}_{+}<0=\widehat{\varphi}_{+}(0)
$$

(see (4.19)), i.e., $\widehat{u}_{+} \neq 0$, so

$$
\widehat{u}_{+}=u_{+}
$$

(see (4.18) and (4.19)).

But $u_{+} \in \operatorname{int} C_{+}$and $\left.\widehat{\varphi}\right|_{W_{+}}=\left.\widehat{\varphi}_{+}\right|_{W_{+}}$. Hence $u_{+}$is a local $C_{0}^{1}(\bar{\Omega})$-minimizer of $\widehat{\varphi}$, thus by virtue of Proposition 2.6, it is also a local $W_{0}^{1, p}(\Omega)$-minimizer of $\widehat{\varphi}$. Similarly, for $v_{-} \in-\operatorname{int} C_{+}$, using this time the functional $\widehat{\varphi}_{-}$. This proves the Claim.

As before (see the proof of Theorem 3.4), without any loss of generality, we may assume that $\widehat{\varphi}\left(v_{-}\right) \leqslant \widehat{\varphi}\left(u_{+}\right)$and because of the Claim, we can find $\varrho \in(0,1)$ small, such that

$$
\widehat{\varphi}\left(v_{-}\right) \leqslant \widehat{\varphi}\left(u_{+}\right)<\inf \left\{\widehat{\varphi}(u):\left\|u-u_{+}\right\|=\varrho\right\}=\widehat{\eta}_{+}, \quad\left\|v_{-}-u_{+}\right\|>\varrho .
$$

(see Aizicovici-Papageorgiou-Staicu [1, proof of Proposition 29] or GasińskiPapageorgiou [20, Theorem 3.4]).

Since $\widehat{\varphi}$ is coercive (see (4.17)), it satisfies the Palais-Smale condition. This fact and (4.20), permit the use of the mountain pass theorem (see Theorem 2.1). So, we can find a solution $y_{0} \in C_{0}^{1}(\bar{\Omega}) \backslash\left\{u_{+}, v_{-}\right\}$of problem (1.1) (see (4.19) and (4.18)). Moreover, as in the proof of Theorem 3.4, using Theorem 2.2, we show that $y_{0} \neq 0$. Since $y_{0} \in\left[v_{-}, u_{+}\right] \cap C_{0}^{1}(\bar{\Omega})$ (see (4.18) and use the nonlinear regularity theory), $y_{0} \notin$ $\left\{u_{+}, v_{-}\right\}$and given the extremality of $u_{+}$and $v_{-}$, we conclude that $y_{0} \in C_{0}^{1}(\bar{\Omega}) \backslash\{0\}$ must be nodal.

Remark 4.5 Compared with the results of Liu-Liu [27, Theorem 1.1] and Liu [28, Theorem 1.2], our work here is more general in many respects. In both the aforementioned works, the differential operator is the $p$-Laplacian (i.e., $G(y)=\frac{1}{p}\|y\|^{p}$ ), $F(z, \cdot) \in C^{1}$, asymptotically at $\pm \infty$ no interaction is allowed with $\widehat{\lambda}_{1}$ and they do not provide sign information for the third solution. However, their condition on $f(z, \cdot)$ near the origin is a little more general than ours, since $f(z, \cdot)$ can be $(p-1)$-linear 
near zero. Here we are forced to assume a "concave" nonlinearity near the origin in order to overcome the nonhomogeneity of the differential operator and produce extremal constant sign solutions and through them produce a nodal solution.

Open Access This article is distributed under the terms of the Creative Commons Attribution Noncommercial License which permits any noncommercial use, distribution, and reproduction in any medium, provided the original author(s) and source are credited.

\section{References}

1. Aizicovici, S., Papageorgiou, N.S., Staicu, V.: Degree theory for operators of monotone type and nonlinear elliptic equations with inequality constraints. Mem. Am. Math. Soc. 196(915), 1-70 (2008)

2. Ambrosetti, A., Lupo, D.: On a class of nonlinear Dirichlet problems with multiple solutions. Nonlinear Anal. 8, 1145-1150 (1984)

3. Ambrosetti, A., Mancini, G.: Sharp nonuniqueness results for some nonlinear problems. Nonlinear Anal. 3, 635-645 (1979)

4. Averna, D., Marano, S., Motreanu, D.: Multiple solutions for a Dirichlet problem with $p$ Laplacian and set-valued nonlinearity. Bull. Aust. Math. Soc. 77, 285-303 (2008)

5. Brézis, H., Nirenberg, L.: $H^{1}$ versus $C^{1}$ local minimizers. C. R. Acad. Sci. Paris Sér. I Math. 317, 465-472 (1993)

6. Chang, K.-C.: Variational methods for nondifferentiable functionals and their applications to partial differential equations. J. Math. Anal. Appl. 80, 102-129 (1981)

7. Cingolani, S., Degiovanni, M.: Nontrivial solutions for $p$-Laplace equations with right hand side having $p$-linear growth at infinity. Comm. Partial Differ. Equ. 30, 1191-1203 (2005)

8. Clarke, F.H.: A new approach to Lagrange multipliers. Math. Oper. Res. 1, 165-174 (1976)

9. Clarke, F.H.: Optimization and Nonsmooth Analysis. Wiley, New York (1983)

10. Corvellec, J.-N.: On the second deformation lemma. Topol. Methods Nonlinear Anal. 17, 55-66 (2001)

11. Diaz, J.I., Saa, J.E.: Existence et unicité de solutions positives pour certaines équations elliptiques quasilinéaires. C. R. Acad. Sci. Paris Sér. I Math. 305, 521-524 (1987)

12. Dunford, N., Schwartz, J.T.: Linear Operators. I. General Theory. Pure and Applied Mathematics, vol. 7. Wiley, New York (1958)

13. Figueiredo, G.M.: Existence of positive solutions for a class of $(p, q)$-elliptic problems with critical growth on $\mathbb{R}^{N}$. J. Math. Anal. Appl. 378, 507-518 (2011)

14. Filippakis, M., Gasiński, L., Papageorgiou, N.S.: Multiplicity result for nonlinear Neumann problems. Can. J. Math. 58, 64-92 (2006)

15. García Azorero, J., Manfredi, J., Peral Alonso, I.: Sobolev versus Hölder local minimizers and global multiplicity for some quasilinear elliptic equations. Commun. Contemp. Math. 2, 385-404 (2000)

16. Gasiński, L., Papageorgiou, N.S.: Multiple solutions for semilinear hemivariational inequalities at resonance. Publ. Math. Debrecen 59,121-146 (2001)

17. Gasiński, L., Papageorgiou, N.S.: A multiplicity result for nonlinear second order periodic equations with nonsmooth potential. Bull. Belg. Math. Soc. Simon Stevin 9, 245-258 (2002)

18. Gasiński, L., Papageorgiou, N.S.: Nonsmooth Critical Point Theory and Nonlinear Boundary Value Problems. Chapman and Hall/ CRC Press, Boca Raton, FL (2005)

19. Gasiński, L., Papageorgiou, N.S.: Nonlinear Analysis. Chapman and Hall/ CRC Press, Boca Raton, FL (2006)

20. Gasiński, L., Papageorgiou, N.S.: Nodal and multiple constant sign solutions for resonant $p$ Laplacian equations with a nonsmooth potential. Nonlinear Anal. 71, 5747-5772 (2009)

21. Guo, Z., Zhang, Z.: $W^{1, p}$ versus $C^{1}$ local minimizers and multiplicity results for quasilinear elliptic equations. J. Math. Anal. Appl. 286, 32-50 (2003)

22. Heikkilä, S., Lakshmikantham, V.: Monotone Iterative Techniques for Discontinuous Nonlinear Differential Equations. Monographs and Textbooks in Pure and Applied Mathematics, Vol. 181. Marcel Dekker, New York (1994)

23. Hu, S., Papageorgiou, N.S.: Handbook of Multivalued Analysis. Volume I: Theory. Mathematics and Its Applications, vol. 419. Kluwer, Dordrecht (1997) 
24. Iannizzotto, A.: Three solutions for a partial differential inclusion via nonsmooth critical point theory. Set-Valued Anal. 19, 311-327 (2011)

25. Ladyzhenskaya, O.A., Uraltseva, N.: Linear and Quasilinear Elliptic Equations. Mathematics in Science and Engineering, vol. 46. Academic Press, New York (1968)

26. Lieberman, G.M.: Boundary regularity for solutions of degenerate elliptic equations. Nonlinear Anal. 12, 1203-1219 (1988)

27. Liu, J.-Q., Liu, S.-B.: The existence of multiple solutions to quasilinear elliptic equations. Bull. Lond. Math. Soc. 37, 592-600 (2005)

28. Liu, S.-B.: Multiple solutions for coercive $p$-Laplacian equations. J. Math. Anal. Appl. 316, 229$236(2006)$

29. Medeiros, E., Perera, K.: Multiplicity of solutions for a quasilinear elliptic problems via the cohomological index. Nonlinear Anal. 71, 3654-3660 (2009)

30. Montenegro, M.: Strong maximum principles for supersolutions of quasilinear elliptic equations. Nonlinear Anal. 37, 431-448 (1999)

31. Motreanu, D., Rădulescu, V.D.: Variational and Non-Variational Methods in Nonlinear Analysis and Boundary Value Problems. Nonconvex Optimization and its Applications, vol. 67. Kluwer, Dordrecht (2003)

32. Naniewicz, Z., Panagiotopoulos, P.D.: Mathematical Theory of Hemivariational Inequalities and Applications. Monographs and Textbooks in Pure and Applied Mathematics, vol. 188. Marcel Dekker, New York (1995)

33. Papageorgiou, E.H., Papageorgiou, N.S.: A multiplicity theorem for problems with the $p$ Laplacian. J. Funct. Anal. 244, 63-77 (2007)

34. Struwe, M.: Variational Methods. Applications to Nonlinear Partial Differential Equations and Hamiltonian Systems, Springer-Verlag, Berlin (1990)

35. Vázquez, J.L.: A strong maximum principle for some quasilinear elliptic equation. Appl. Math. Optim. 12, 191-202 (1984)

36. Zhang, Q.: A strong maximum principle for differential equations with nonstandard $p(x)$-growth condition. J. Math. Anal. Appl. 312, 24-32 (2005) 